\title{
Unusual Aorto-mitral Discontinuity Caused by Infective Endocarditis
}

\author{
Tamami Nakagawa ${ }^{1}$, Mika Mori ${ }^{1}$, Hayato Tada ${ }^{1}$, Miho Ohira ${ }^{1}$, Kenji Iino ${ }^{2}$, Kenshi Hayashi ${ }^{1}$, \\ Hirofumi Takemura ${ }^{2}$, Masakazu Yamagishi ${ }^{1}$ and Masa-aki Kawashiri ${ }^{1}$
}

\begin{abstract}
:
Aorto-mitral discontinuity is considered a relatively rare complication of infective endocarditis, usually requiring invasive surgical procedures, including double valve replacement in addition to aortic root replacement/repair. In the present case report, images were produced using transthoracic echocardiograms and transesophageal echocardiograms, which may assist in the planning of surgical procedures. The images captured using real-time three-dimensional transesophageal echocardiograms revealed aorto-mitral discontinuity and a perforated mitral valve aneurysm of the anterior leaflet.
\end{abstract}

Key words: infective endocarditis, transthoracic echocardiogram, transesophageal echocardiogram

(Intern Med 58: 2373-2376, 2019)

(DOI: 10.2169/internalmedicine.2629-19)

\section{Introduction}

Aorto-mitral discontinuity is a relatively rare critical complication associated with infective endocarditis (IE). We herein report a case with unusual aorto-mitral discontinuity caused by IE, which was identified using transthoracic echocardiograms (TTEs) as well as transesophageal echocardiograms (TEEs), including real-time three-dimensional transesophageal echocardiogram (RT3D TEE). Through this approach, we detected aorto-mitral discontinuity associated with anterior mitral valve aneurysm perforation.

\section{Case Report}

A 75-year-old man complicated with edema of the lower limbs was admitted to his previous doctor. He had no apparent medical history, including hypertension and diabetes, nor any predisposing valuvular heart diseases. TTEs showed aortic regurgitation (AR) and mitral regurgitation (MR) with a massive structure, and the left ventricular dimension at the diastolic phase was determined to be $57 \mathrm{~mm}$, with the ejection fraction preserved at the time (estimated at $66 \%$ by the Teichholz method). At that point, no perforation was ob- served, including for the mitral valve, and his aorto-mitral continuity was preserved.

The laboratory data revealed an elevated level of Creactive protein (CRP) at $6.1 \mathrm{mg} / \mathrm{dL}$, and Streptococcus species were detected in the patient's blood culture. A diagnosis of IE with heart failure was made, and the patient received antibiotic therapy (penicillin) for six weeks. His serum CRP level decreased to within the normal range $(0.2 \mathrm{mg} / \mathrm{dL})$, and his blood culture became negatively associated with antibiotic therapy. However, his dyspnea on effort worsened gradually, despite medical treatment, and he was referred to Kanazawa University Hospital based on a surgical indication for MR and AR. At that time, he was conscious and alert with a body temperature of $36.8^{\circ} \mathrm{C}$, heart rate of 74 beats/ min, blood pressure of $113 / 50 \mathrm{mmHg}$, and percutaneous oxygen saturation of $97 \%$ on room air. Pansystolic heart murmur at his apex (Levine III/VI) as well as diastolic heart murmur at the third left sternal border (Levine III/VI) and mild edematous changes were observed at his lower limbs, although no apparent coarse crackles were audible in the bilateral lung fields.

Chest X-ray showed cardiomegaly and pulmonary congestion despite medical treatments using torasemide $(4 \mathrm{mg})$ and tolvaptan (7.5 mg) (Fig. 1A). TTEs examined at our institute

${ }^{1}$ Division of Cardiovascular and Internal Medicine, Kanazawa University Graduate School of Medical Sciences, Japan and ${ }^{2}$ Department of Thoracic, Cardiovascular and General Surgery, Kanazawa University Graduate School of Medicine, Japan

Received: January 9, 2019; Accepted: February 24, 2019; Advance Publication by J-STAGE: May 22, 2019

Correspondence to Dr. Hayato Tada, ht240z@med.kanazawa-u.ac.jp 

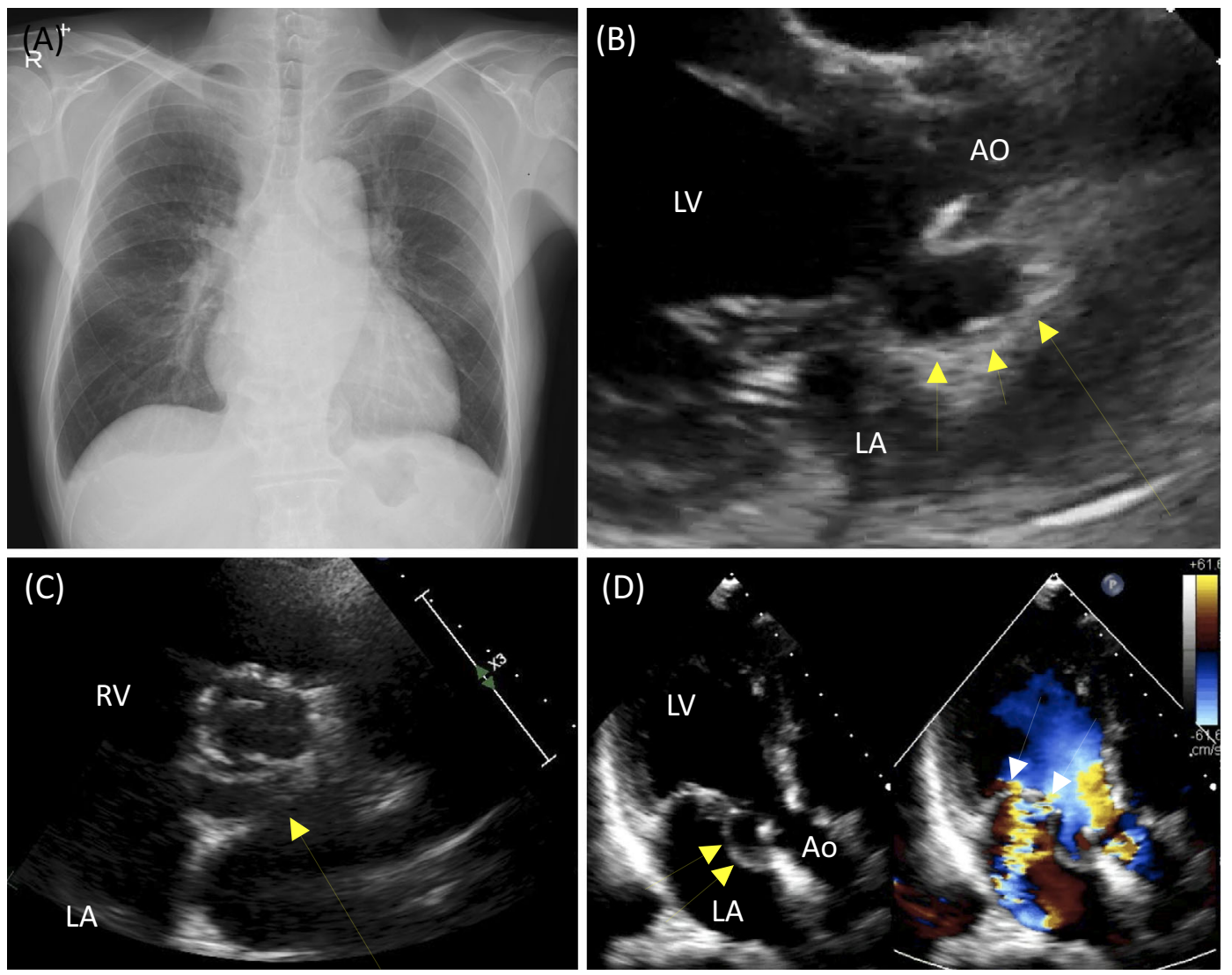

Figure 1. TTE and chest X-ray findings of a patient with aorto-mitral discontinuity caused by infective endocarditis. (A) Chest X-ray demonstrating cardiomegaly with mild pulmonary congestion. (B) Parasternal long-axis view demonstrating aneurysmal change of aorto-mitral discontinuity (yellow arrowheads). (C) Left panel of parasternal short-axis view demonstrating aneurysmal change of aorto-mitral discontinuity (yellow arrowheads). (D) Left panel of apical long-axis view demonstrating aneurysmal change of aorto-mitral discontinuity (yellow arrowheads). Right panel of apical long-axis view with color Doppler demonstrating mitral valve aneurysm of the anterior leaflet and severe mitral regurgitation (white arrowheads). TTE: transthoracic echocardiography, AO: aorta, LV: left ventricle, $L A$ : left atrium

with a parasternal long-axis and short-axis view showed aorto-mitral discontinuity and protrusion to the left atrium (Fig. 1B and C). In addition, a TTE with an apical long-axis view revealed aneurysmal changes of aorto-mitral discontinuity associated with severe MR (Fig. 1D). The left ventricular dimension at the diastolic phase was unchanged from the initial evaluation $(59 \mathrm{~mm})$, and the ejection fraction was still preserved (estimated at $65 \%$ by the Teichholz method). A TEE with a $135^{\circ}$ view showed that the fibrous structures had been transected, and the front of the left atrium was protruding inwards, resulting in the appearance of the low-echoic space (Fig. 2A). Severe AR flow had affected part of the aorto-mitral continuity, causing the aneurysmal change and perforation of the anterior mitral valve (Fig. 2B). An RT3D TEE showed anterior mitral valve aneurysm and perforation as well as an area of aorto-mitral discontinuity connecting with the left ventricle (Fig. 3).

The patient successfully underwent aortic valve replacement with a 19-mm Carpentier-Edwards PERIMOUNT Magna EASE aortic heart valve (CarpentierEdwards, Irvine,
USA), and mitral valve replacement with a $25-\mathrm{mm}$ St. Jude Medical Epic valve (St. Jude Medical, St. Paul, USA) associated with anterior mitral commissure (AMC) plasty. Intraoperative findings revealed aorto-mitral discontinuity (Fig. 4A) and a perforated mitral valve aneurysm of the anterior leaflet (Fig. 4B). The histology of all valves indicated myxoid degeneration. In addition, aneurysmal change with a perforation was observed at the middle part of the anterior mitral leaflet, caused by IE (Fig. 4C). The micropathological findings of the excised aortic and mitral valve revealed infiltrations of lymphocytes, plasma cells, histiocytes, and a small amount of eosinophils, findings that were compatible with changes caused by IE, although no bacterial bodies were detected.

The clinical course subsequent to the surgical procedure was good, and the patient was discharged 26 days after surgery. The patient was followed without complications at our outpatient department. 


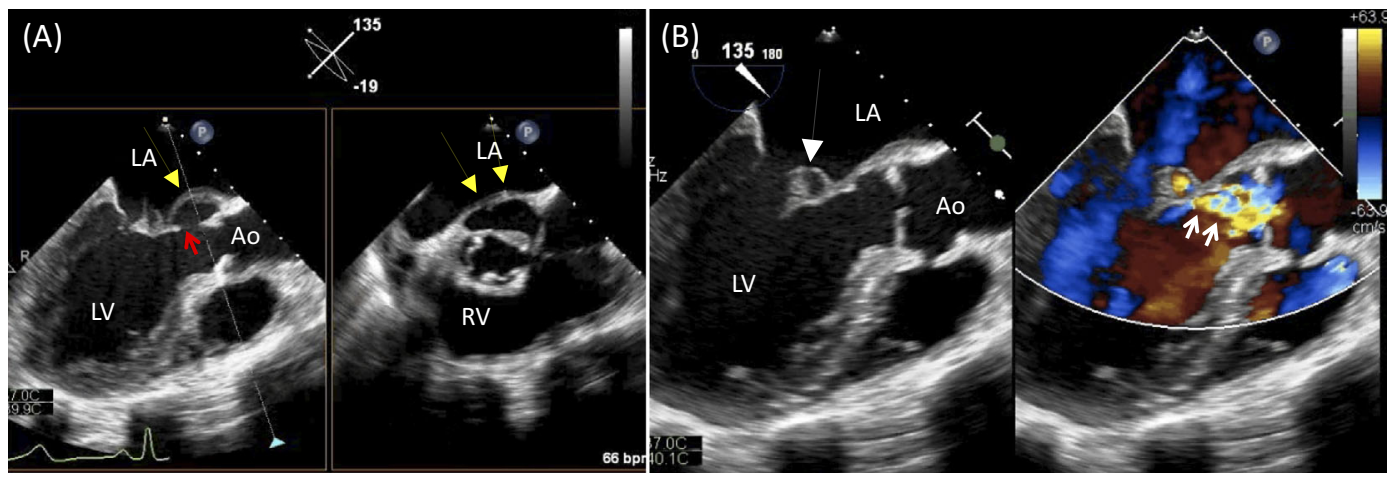

Figure 2. TEE findings of a patient with aorto-mitral discontinuity caused by infective endocarditis. (A) Left panel of long-axis view and right panel of short-axis view showing that the fibrous structure around the vestibule of the left atrium protruded inwards, inducing aneurysmal change of the aorto-mitral discontinuity (yellow arrowheads). (B) Left panel of long-axis view demonstrating perforated mitral valve aneurysm of the anterior leaflet (white arrowhead). Right panel of long-axis view in color Doppler demonstrating severe aortic regurgitation with prolapse toward the aorto-mitral continuity and the mitral valve aneurysm. TEE: transesophageal echocardiography, AO: aorta, LV: left ventricle, $L A$ : left atrium

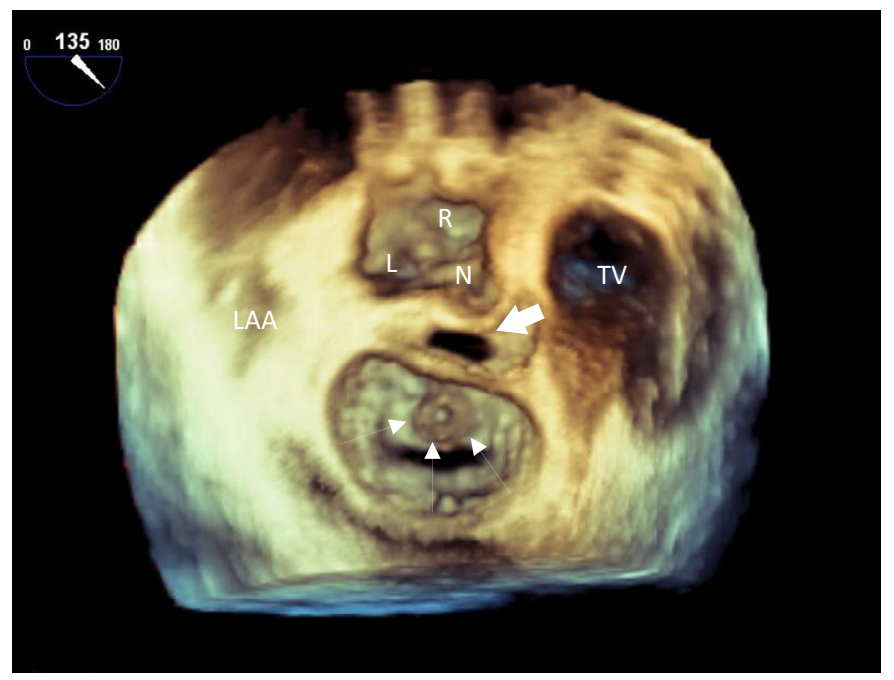

Figure 3. RT3D TEE findings of a patient with aorto-mitral discontinuity caused by infective endocarditis. RT3D TEE shows anterior mitral leaflet aneurysm with perforation. The lumen of aorto-mitral discontinuity is open to the LV. RT3D TEE: real-time three-dimensional transesophageal echocardiography, LAA: left atrial appendage, R: right coronary cusp, L: left coronary cusp, N: non-coronary cusp, TV: tricuspid valve

\section{Discussion}

In the case described here, severe AR flow affected part of aorto-mitral junction, leading to aorto-mitral discontinuity and aneurysmal change of the anterior mitral valve. It is of note that he had no apparent medical history, including hypertension and diabetes, nor any valvular heart diseases. In this regard, it has been shown that a substantial proportion of patients with IE did not have any predisposing cardiac diseases (1). In addition, our patient was treated appropriately according to the current Japanese guideline [Guidelines for Prevention and Treatment of Infective Endocarditis (JCS 2017)]. It is therefore unclear why the infection in the present case grew so severe. We would like to emphasize that even individuals without predisposing cardiac diseases can be complicated with severe valvular heart disease associated with IE.

The prevalence of aorto-mitral discontinuity has been shown to be as high as $10.6 \%$ among cases of left-sided 

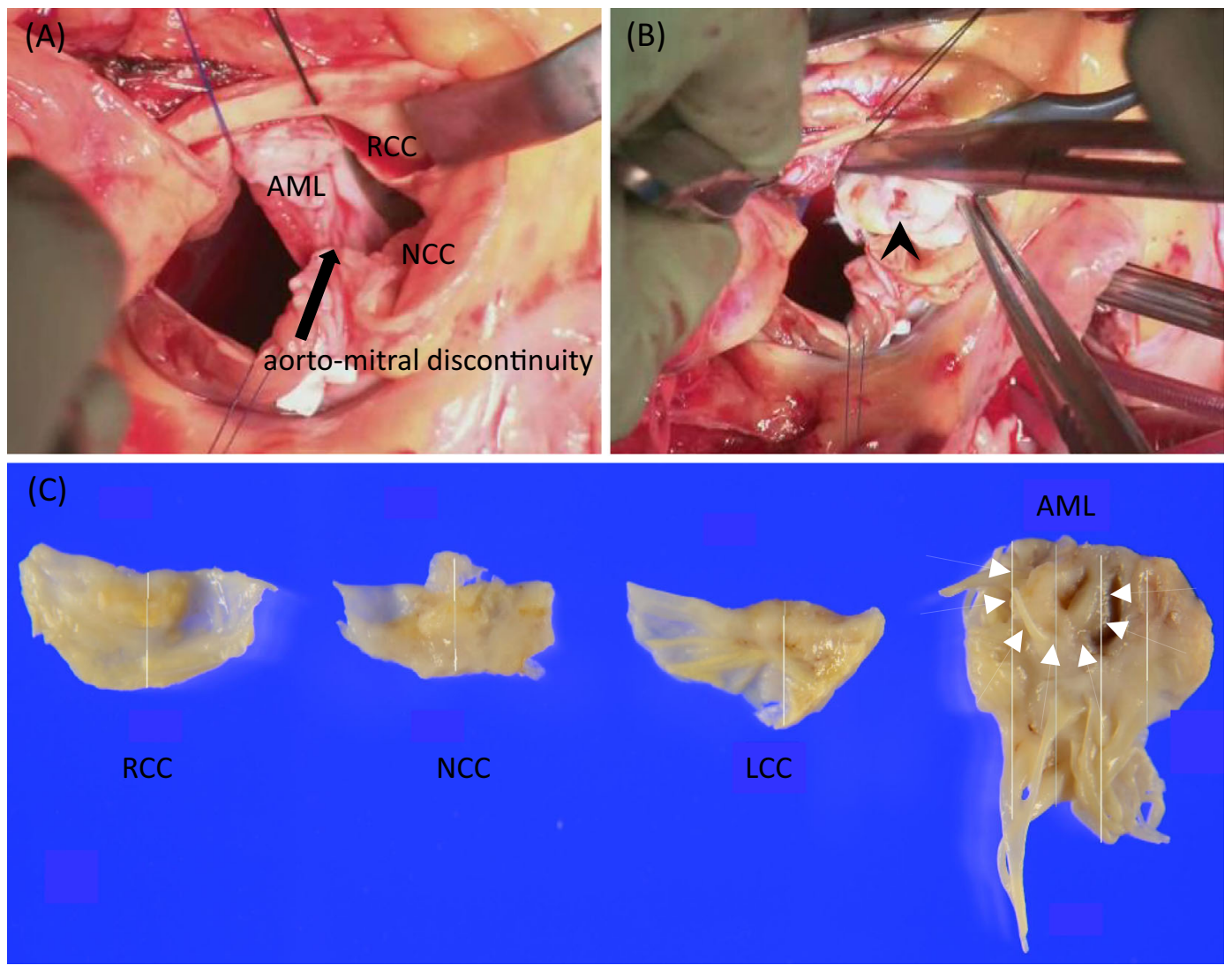

Figure 4. Findings during the surgical procedure. (A) Intraoperative findings demonstrating aortomitral discontinuity (black arrow). (B) Intraoperative findings demonstrating perforated mitral valve aneurysm of the anterior leaflet (black arrowhead). (C) RCC, NCC, LCC, and AML are shown in the order of left to right. Histologically, all of these valves showed myxoid degeneration. In addition, aneurysmal change with perforation was observed in the middle part of the anterior mitral leaflet caused by infective endocarditis (white arrowheads). RCC: right coronary cusp, LCC: left coronary cusp, NCC: non coronary cusp, AML: anterior mitral leaflet

IE (2), and in left-sided double-valve IE, aorto-mitral discontinuity reconstruction is required in $28 \%$ of cases (3). Surgical treatment should always be considered in cases of IE, as more than half of patients with IE have been surgically treated (4), although the extent and complexity of the surgical procedure have been shown to be associated with a poor prognosis, with an early mortality rate ranging between $10 \%$ and $32 \%(5,6)$. It is important to be aware of cases complicated with aorto-mitral discontinuity that require additional surgical procedures among patients with IE, particularly those with left-sided double-valve IE.

The authors state that they have no Conflict of Interest (COI).

\section{References}

1. Nakatani S, Mitsutake K, Hozumi T, et al. Current characteristics of infective endocarditis in Japan: an analysis of 848 cases in 2000 and 2001. Circ J 67: 901-905, 2003.
2. Forteza A, Centeno J, Ospina V, et al. Outcomes in aortic and mitral valve replacement with intervalvular fibrous body reconstruction. Ann Thorac Cardiovasc Surg 99: 838-845, 2015.

3. Sheikh AM, Elhenawy AM, Maganti M, Armstrong S, David TE, Feindel CM. Outcomes of double valve surgery for active infective endocarditis. J Thorac Cardiovasc Surg 138: 69-75, 2009.

4. Nakatani S, Mitsutake K, Ohara T, Kokubo Y, Yamamoto H, Hanai S; CADRE Investigators. Recent picture of infective endocarditis in Japan--lessons from Cardiac Disease Registration (CADRE-IE). Circ J 77: 1558-1564, 2013.

5. Olaison L, Pettersson G. Current best practices and guidelines. Indications for surgical intervention in infective endocarditis. Cardiol Clin 21: 235-251, 2003.

6. Forteza A, Centeno J, Ospina V, et al. Outcomes in aortic and mitral valve replacement with intervalvular fibrous body reconstruction. Ann Thorac Surg 99: 838-845, 2015.

The Internal Medicine is an Open Access journal distributed under the Creative Commons Attribution-NonCommercial-NoDerivatives 4.0 International License. To view the details of this license, please visit (https://creativecommons.org/licenses/ by-nc-nd/4.0/).

(C) 2019 The Japanese Society of Internal Medicine Intern Med 58: 2373-2376, 2019 